\title{
Place de la matière azotée dans le mécanisme de la coagulation présure du lait
}

\author{
A.J. ILBOUDO ${ }^{1 *}$, A. SAVADOGO ${ }^{1}$, M.G. SEYDI $^{2}$ et A.S. TRAORE ${ }^{1}$ \\ ${ }^{1}$ Université de Ouagadougou, Centre de Recherche en Sciences Biologiques Alimentaires et Nutritionnelles \\ (CRSBAN) 03 BP 7021 Ouagadougou 03, Burkina Faso. \\ ${ }^{2}$ Ecole Inter-Etats des Sciences et Médecine Vétérinaires de Dakar, B.P.5077 Dakar Fann, Sénégal. \\ *Auteur correspondant; E-mail : ilboudo_jules@hotmail.fr; andre_ilboudo@univ-ouaga.bf
}

\section{RESUME}

Les protéines jouent un rôle très important dans le processus de gélification du lait. Parmi ces protéines du lait, les caséines constituent la fraction majeure et la plus déterminante en matière de technologie laitière. Les caséines se présentent sous la forme de micelles formées par l'association de différents constituants parmi lesquels on cite: les caséines $\alpha_{\mathrm{s} 1}$ et $\alpha_{\mathrm{s} 2}$, la caséine $\beta$, la caséine $\kappa$ et des minéraux, notamment du calcium et du phosphate. La caséine $\kappa$, bien que non majoritaire dans la micelle, 3,3 $\mathrm{g} \mathrm{l}^{-1}$, est la protéine laitière qui détient le rôle clef dans la coagulation du lait par la présure. Elle comporte deux variantes génétiques, la caséine $\kappa$ A qui est la plus fréquemment rencontrée dans le lait (75-85\%) des cas et la caséine $\kappa$ B (15-25\%) des cas. Les micelles de caséine peuvent être déstabilisées par une acidification ou par l'action de certaines enzymes protéolytiques comme la présure. Cette déstabilisation entraîne la coagulation du lait. C'est ainsi qu'une addition de présure au lait provoque sa coagulation par hydrolyse de la caséine $\kappa$ située en périphérie de la micelle. Cette hydrolyse scinde le lien 105-106 (lien Phe-Met) et libère le glycomacropeptide qui est la partie hydrophile de la $\kappa$-caséine chargée négativement et responsable des répulsions électrostatiques. La coagulation du lait par la présure est influencée par plusieurs facteurs parmi lesquels on cite la concentration en enzyme, la température, le $\mathrm{pH}$ et la concentration en ions $\mathrm{Ca}^{2+}$. L'objectif de ce travail est de faire une revue des connaissances sur les protéines du lait et leurs rôles dans le mécanisme de la coagulation laitière.

(C) 2012 International Formulae Group. All rights reserved.

Mots clés: Lait, protéines, coagulation, caséine, présure.

\section{INTRODUCTION}

La formation d'un gel ou gélification est une propriété très importante des macromolécules que sont les protéines et les polyosides dans un système alimentaire (Cayot, Lorient, 1998 ; Mahaut et al., 2000 ; Fernandez et al., 2009; Bonfatti et al., 2010 ; Hallen et al., 2010). Le processus de coagulation est très utilisé en technologie laitière et conduit à l'obtention de produits variés (yaourt, lait caillé, fromage). Le démarrage de ce processus est consécutif à la présence dans le lait de bactéries lactiques dont la principale fonction est la dégradation du lactose en acide lactique. L'apparition de l'acide lactique entraine la chute $\mathrm{du} \mathrm{pH}$. Lorsque celui-ci atteint la valeur seuil de 4,5 correspondants au point isoélectrique de la caséine, la coagulation se déclenche suite à l'agrégation et la précipitation des micelles de 
caséines (Zbikowska et al., 2004). Le processus peut aussi être l'œuvre d'une enzyme la présure qui a la propriété de coaguler le complexe caséine. Dans ce cas la coagulation est la conséquence de la transformation du phosphocaséinate de calcium à l'état soluble dans le lait, sous l'action de la présure en phosphoparacaséinate insoluble (Dalgleish, 1998; Cayot, Lorient, 1998 ; Pires et al., 1999). Les progrès acquis dans la connaissance scientifique et technologique des constituants du lait et de leurs propriétés, plus particulièrement les protéines, ont incité les acteurs de la filière lait à mieux valoriser l'énorme potentiel nutritionnel et techno fonctionnel du lait (Cayot, Lorient, 1998). En effet les propriétés fonctionnelles de ces protéines en font des auxiliaires technologiques de choix ou même des substituts d'autres protéines (œuf par exemple) de coût plus élevé.

Le processus de gélification a aussi été abordé dans d'autres travaux notamment ceux de Brulé et al. (1997), Hallen et al. (2010) qui ont travaillé sur la micelle de caséine et la coagulation du lait. Il en est de même des travaux de Walstra et al. (1999) dont les travaux ont porté sur «Principles of Milk Properties and Processes » et de ceux d'Amiot et al. (2002) dont le travail a porté sur Composition, propriétés physicochimiques, valeur nutritive, qualité technologique et techniques d'analyse du lait. Tous ces travaux font ressortir le rôle essentiel de la caséine dans ce processus. Ils montrent en outre que ce processus se décompose en trois grandes étapes : hydrolyse enzymatique de la caséine $\kappa$; agrégation des micelles de caséine déstabilisées ; développement d'un réseau par réticulation et formation de gel.

Le taux protéique du lait est celui qui influence le plus le processus de gélification et les caractéristiques rhéologiques du caillé (Lopez et al., 1998; Vignola, 2002; Vliet et al., 2004). Dans le lait la teneur en protéines varie entre 32 et $35 \mathrm{~g} \mathrm{l}^{-1}$ (Mahaut et al., 2000 ; Vignola, 2002 ; Bonfati, 2010). Les protéines du lait sont classées en deux catégories selon leur solubilité dans l'eau et leur stabilité. Ainsi, on distingue les caséines qui forment une suspension colloïdale dans la phase aqueuse du lait et les protéines du lactosérum dites protéines solubles ou protéines du petit lait (Fernandez et al., 2009). Une suspension colloïdale est un mélange constitué d'une phase dispersée solide non solubilisée, présente sous forme de très fines particules solides dans une phase dispersante liquide. Lorsque les particules ont beaucoup d'affinité pour la phase aqueuse, on nomme ce système une solution colloïdale (Amiot et al., 2002).

Cet article de synthèse a pour objectif une meilleure connaissance des protéines du lait impliquées dans le processus de coagulation du lait par la présure pour une maîtrise de la technologie laitière par les professionnels de la filière dans les zones périurbaines des grandes villes Ouest Africaines.

\section{Généralités sur les matières azotées du lait}

La teneur moyenne en protéines d'un lait normal est d'environ $3,2 \%$, ce qui représente $95 \%$ de l'azote total de ce lait. Les autres $5 \%$ sont formés par la matière azotée non protéique (urée, créatine, créatinine, acides aminés, petits peptides, ammoniac) (Mathieu, 1985; Cayot et Lorient, 1998 ; Mahaut et al., 2000). Environ $80 \%$ des protéines du lait sont constituées de caséines qui précipitent à $\mathrm{pH} 4,6$ et forment la matrice fromagère, les $20 \%$ restants forment les protéines du lactosérum qui sont solubles à toutes les valeurs de $\mathrm{pH}$ si elles ne sont pas dénaturées (Visser et al., 1980; Brulé et al., 1997).

Les protéines du lait existent sous un grand nombre de structures différentes. Elles peuvent être toutefois subdivisées en deux grandes catégories, qui correspondent, à deux 
formes structurales très dissemblables (Alais, 1984; Fernandez et al., 2009). La première catégorie est constituée par les protéines solubles, dites protéines du lactosérum, qui ne précipitent pas lors de la coagulation enzymatique du lait, ou lors d'une acidification. La deuxième est formée des caséines. La caséine représente la fraction majeure des protéines du lait. Ce sont des éléments essentiels au bon fonctionnement des cellules vivantes. La présence de groupements phosphoséryls confère aux caséines une très grande affinité vis-à-vis du calcium, du magnésium et des oligoéléments (Dickson et Perkins, 1971).

\section{Place des protéines dans la coagulation du lait}

Les protéines du lait sont classées en deux catégories selon leur solubilité dans l'eau et leur stabilité. Ainsi, on distingue d'un côté les différentes caséines qui sont en suspension colloïdale dans la phase aqueuse du lait et les protéines du lactosérum dites protéines solubles ou protéines du petit lait. Les caséines sont celles qui sont impliquées dans le processus de gélification du lait (Bonfatti et al., 2010).

\section{Les caséines}

Les caséines constituent la fraction majeure des protéines du lait (Figure 1). Cette fraction est la plus déterminante en matière de technologie fromagère. Elles se trouvent sous forme de micelles. Ces micelles précipitent sous l'action de la présure ou lors d'une acidification à un pH d'environ 4,6.

\section{Structure et organisation de la micelle de caséine \\ La micelle de caséine a une forme sphéroïdale d'un diamètre variant habituellement de 80 à $600 \mathrm{~nm}$, selon une répartition monomodale, avec une fréquence maximum au diamètre de $177 \mathrm{~nm}$}

(Ghazarossian Hazarossian, 1996). Plusieurs modèles d'organisation des micelles de caséine ont été décrits (Schmidt, 1982; Walstra et Jenness, 1992; Holt, 1992; Holt, Horne, 1996; McMahon et McManus, 1998). Le modèle à sous-unités proposé par Schmidt, (1980, 1982) s'appuie sur des observations en microscopie électronique (Walstra et Jenness, 1992; Walstra, 1999). La micelle est constituée d'un ensemble de sous-unités de nature exclusivement protéique et de composition variable. Ces sous-unités sont associées les unes aux autres par des interactions de type hydrophobe, électrostatique et hydrogène et par des clusters de phosphate de calcium.

Un autre modèle, à structure ouverte a été proposé par Holt et Horne (1996) (Figure 2). En A, la structure ouverte présente des chaînes polypeptidiques reliées par des nanoclusters de phosphate de calcium dans le cœur et une couche chevelue constituée de segments de faible densité à la périphérie. En $\mathbf{B}$, les détails des associations des caséines par interaction entre les régions hydrophobes (rectangles noirs) et par liaison des régions hydrophiles contenant des résidus phosphosérine aux clusters de phosphate de calcium colloïdal (Horne, 1998).

Dans ce modèle, la micelle de caséine se présente comme un édifice poreux constitué d'un réseau de caséines $\alpha_{\mathrm{s}}$ et notamment de caséine $\alpha_{\mathrm{s} 1}$. Cet édifice est stabilisé par le phosphate de calcium colloïdal et la caséine $\kappa$ (Holt et Horne, 1996; Horne, 1998). Le calcium et le phosphate sont associés sous forme de phosphate de calcium colloïdal. Etant donné le pouvoir séquestrant des résidus phosphosérine des caséines, le rapport $\mathrm{Ca}+\mathrm{Mg} / \mathrm{P}$ inorganique est de 1,58. De ce fait, le phosphate de calcium colloïdal serait à l'état de sels tricalciques amorphes $\mathrm{Ca}_{9}\left(\mathrm{PO}_{4}\right)_{6}$ (Pyne et McGann, 1960; Schmidt, 1980, 1982). Mais cette hypothèse de phosphate tricalcique n'est pas admise par 
tous les auteurs. Holt (1997) a en effet estimé que le phosphate de calcium micellaire ressemblerait à une brurshite (phosphate dicalcique) modifiée dont la composition serait $\mathrm{Ca}\left(\mathrm{HPO}_{4}{ }^{2-}\right)_{0,7}\left(\mathrm{PO}_{4}{ }^{3-}\right)_{0,2}\left(\mathrm{H}_{2} \mathrm{O}\right)_{\mathrm{x}}$. Van Dijk (1990) propose quant à lui un modèle de phosphate colloïdal correspondant à des amas d'ions de taille différente dont le principal serait constitué par 2 groupements phosphosérine de 2 chaînes caséiques différentes, 4 ions phosphate inorganique et 8 cations divalents dont six ions calcium.

Les particules de phosphate de calcium sont réparties dans la matrice micellaire. Un modèle de nanoclusters de phosphate de calcium comprenant un cœur sphérique constitué de phosphate de calcium hydraté amorphe (environ 355 unités de $\mathrm{CaHPO}_{4}, 2 \mathrm{H}_{2} \mathrm{O}$ ) de rayon 2,3 $\mathrm{nm}$ entouré par 49 chaînes phosphopeptidiques est également décrit (Little et Holt, 2004).

\section{Composition de la micelle de caséine}

Une micelle de caséine est formée par l'association des caséines $\alpha_{\mathrm{s} 1}, \alpha_{\mathrm{s} 2}$, caséine $\beta$, caséine $\kappa$ et des minéraux, notamment le calcium et le phosphate. La micelle contient 20000 à 150000 molécules de caséines (Schmidt, 1980). Les proportions moyennes des différents constituants de la micelle de caséine sont présentées dans le Tableau 1.

\section{Propriétés physicochimiques des micelles de caséine}

Le diamètre moyen de la micelle est compris entre 180 et $200 \mathrm{~nm}$ (McGann et al., 1980; Schmidt, 1982; Holt,1992; Dalgleish, 1998). Sa voluminosité varie entre 1,5 et 7,1 mL/g (McMahon et al., 1984a; Walstra, 1999 ) et son potentiel de surface entre -15 et $-20 \mathrm{mV}$.

Compte tenu de leur dimension, les micelles de caséine présentent une grande stabilité en raison de leur charge nette et de leur degré d'hydratation. La charge négative de la surface de la micelle est en partie responsable des répulsions électrostatiques entre micelles et contribue au maintien de la dispersion malgré la forte densité de population des micelles et la faible distance entre les particules. Les micelles fixent une grande proportion d'eau $(3,7 \mathrm{~g} / \mathrm{g}$ de protéine) qui est immobilisée à la surface et forme une enveloppe d'hydratation qui protège et stabilise la micelle.

Ces différents facteurs de stabilité des micelles dépendent de divers paramètres liés à la composition saline de la phase aqueuse (concentration en $\mathrm{H}^{+}, \mathrm{Ca}^{2+}$, phosphate, citrate) ou à la composition même des micelles (proportions relatives des caséines, teneurs en phosphate de calcium). L'acidification ou l'action de certaines enzymes protéolytiques peuvent être à l'origine de la déstabilisation des micelles de caséine et entraîner la coagulation du lait voire sa floculation si l'acidification est brutale (Holt et Horne, 1996).

Ainsi, l'agrégation des micelles peut être obtenue de plusieurs manières :

- après modification physique ou chimique irréversible et destruction ou fusion de la micelle. C'est ce qui se produit lors de l'acidification rapide $(\mathrm{pH} \quad 4,6)$ où les monomères précipitent avec fuite du phosphate de calcium, ou lors de l'action de cations à forte concentration et d'éthanol (Holt, 1997 ; Cayot et Lorient, 1998).

Même une légère acidification modifie suffisamment la structure micellaire pour que les caséines deviennent instable à la chaleur (Zbikowska et al., 2004; Chandrapala et al., 2010). Lorsque le pH passe de 6,7 à 5,5, les charges négatives présentes à la surface des micelles de caséine sont neutralisées ce qui produit une augmentation du diamètre moyen des micelles causée par l'agglomération des petites micelles sur les grosses. Ce diamètre moyen passe de $180 \mathrm{~nm}$ à $1300 \mathrm{~nm}$. Si l'acidification se poursuit jusqu'au point 
isoélectrique de la caséine soit un pH de 4,65, les micelles perdent complètement leur structure par dissolution totale du calcium micellaire. Dès lors, les caséines sont solubilisées et perdent leur propriété de suspension colloïdale. Il y a alors étirement puis enchevêtrement des protéines avec formation d'un gel (Visser et al., 1980 ; Dalgleish et Law, 1989 ; Le Graet et Brule, 1993 ; Vignola, 2002).

- la température du lait influence aussi la vitesse d'agrégation des protéines (McMahon et al., 1984a; Walstra, 1999; Kethireddipalli et al., 2010). Des traitements thermiques de l'ordre de $60{ }^{\circ} \mathrm{C}$ sont à l'origine d'une modification de la structure des protéines. Cette modification étant réversible, il y a peu de d'influence sur les propriétés physicochimiques des protéines. Cependant, lors d'un chauffage à une température de 90 ${ }^{\circ} \mathrm{C}$, la $\beta$-lactoglobuline se dénature et se fixe sur la micelle de caséine par un pont disulfure $(-\mathrm{S}-\mathrm{S})$ entre cette protéine et la caséine $\kappa$ (Jean et al., 2006). Cette fixation augmente le volume des micelles de caséine ce qui augmente la viscosité du produit laitier (Singh et Waungana, 2001; Vignola, 2002). Les hautes températures peuvent aussi entraîner une agrégation irréversible des micelles de caséine après modification de la surface micellaire rendue hydrophobe (Cayot et Lorient, 1998; Kethireddipalli et al., 2010; Chandrapala et al., 2010; Jean et al., 2006).

- l'agrégation des micelles peut être enfin obtenue par action de la chymosine. L'addition de présure au lait provoque sa coagulation par hydrolyse de la caséine $\kappa$ située en périphérie de la micelle. Cette hydrolyse scinde le lien 105-106 (lien PheMet) et libère le glycomacropeptide qui est la partie hydrophile de la $\kappa$-caséine chargée négativement et responsable des répulsions électrostatiques (Mahaut et al., 2000; Vignola, 2002; Hemar et al., 2004; Kumar et al., 2006).
Rôle de la caséine dans le processus de gélification

La proportion relative entre les différentes caséines, à concentration protéique constante, influence considérablement l'aptitude à la coagulation (acide ou par la chymosine) du lait. Un lait riche en caséine $\beta$, par exemple, donne un gel présure plus ferme (Remeuf et al., 1991).

La taille de la micelle influence aussi considérablement les capacités fromagères d'un lait. Cette taille semble parfaitement corrélée avec la teneur en caséine $к$. Ainsi, la teneur en caséine $\kappa$ et la taille des micelles apparaissent comme des paramètres essentiels dans les aptitudes fromagères d'un lait (McNeill et Donnely, 1987; Remeuf et al., 1991; Hallen et al., 2010).

La caséine $\kappa$ constitue «la chevelure» stabilisante de la micelle. Bien que non majoritaire dans la micelle, 3,3 g. l $^{-1}$, la caséine $\kappa$ est la protéine laitière qui détient un rôle clef dans la coagulation du lait par la présure (Ribadeau-Dumas et Grappin, 1989; Hallen et al., 2010). Elle comporte deux variantes génétiques, la caséine $\kappa \mathrm{A}$ qui est la plus fréquemment rencontrée dans le lait $(75-85 \%$ des cas) et la caséine $\kappa$ B $(15-25 \%$ des cas) (Remeuf et al., 1991). Le variant B de la caséine $\kappa(\kappa-\mathrm{CNB})$ diffère du variant $\mathrm{A}(\kappa-$ CNA) de deux résidus d'acides aminés: le résidu isoleucyle à la place du résidu thréonyle 136 et le résidu alanyle à la place du résidu aspartyle 148 (Mercier et al., 1973; Farrell et al., 1993). La liaison peptidique 105-106 entre le résidu phénylalanyle et le résidu méthionyle est la liaison hydrolysée spécifiquement par la chymosine après l'emprésurage du lait.

La caséine $\kappa$ est, après la caséine $\beta$, la plus hydrophobe des caséines. L'hydrolyse par la chymosine donne une glycomacropeptide (106-169) de nature très hydrophile ce qui donne un caractère très amphiphile à la protéine (Swaisgood, 1982). 
La partie N-terminale (1-105) est légèrement cationique et très hydrophobe. Au moment de l'hydrolyse par la chymosine, la para caséine $\kappa(1-105)$ reste accroché à la micelle et celle-ci, en perdant son pôle hydrophile stabilisant, précipite (Cayot et Lorient, 1998; Hallen et al., 2010).

\section{Mécanisme de la coagulation enzymatique du lait}

Un certain nombre d'enzymes d'origine animale, végétale et microbienne ont la propriété de coaguler le lait. Les enzymes d'origine animale sont représentées essentiellement par la présure de veau, d'agneau ou de chevreau et la pepsine de porc ou de poulet (Dalgleish, 1992). Pour les enzymes d'origine végétale, on cite la ficine (figuier), la bromélaïne (ananas) et les enzymes de la pomme de sodome (Calotropis procera (Aworh et Muller, 1987), d'artichaut, de chardon, et de courge (Aworh et Muller, 1987) Enfin certains microorganismes sont capables de synthétiser un certain nombre d'enzymes utilisées pour coaguler le lait. Parmi ceux-ci, des moisissures (Endothia parasitica, Mucor meihei, Mucor pusullus, Mucor baciliformis etc), des bactéries (Escherichia coli, Bacillus subtilis, Bacillus cereus etc.) et des actinomycètes (Nocardiopsis sp) (Arima et al., 1967 ; Sardinas,1968 ; Arima et al., 1970; Martin et al., 1980; Aworh et Muller,1987; Areces et al., 1992; Kees Marlies, 1996; Cavalcanti et al., 2004). Dans la présente synthèse nous nous limiterons au mode d'action de la présure.

\section{La présure}

La présure est un mélange de chymosine et de pepsine. C'est un extrait liquide ou pâteux provenant des caillettes des jeunes ruminants (Mahaut et al., 2000; D'Ambrosio et al., 2003; Anema et al., 2005).
Selon la législation française, l'extrait de présure doit contenir au minimum $520 \mathrm{mg}$ de chymosine active par litre et le rapport chymosine/pepsine doit être supérieur ou égal à 1,38 , ce qui signifie que 75 à $80 \%$ de l'activité coagulante est due à la chymosine. La chymosine est donc l'enzyme majeure de la coagulation du lait (Dalgleish, 1992; Walstra et Jenness, 1992). Elle appartient à la famille des endopeptidases, protéases agissant à l'intérieur des chaînes polypeptidiques. Elle possède une activité très spécifique. En effet, elle n'hydrolyse que la caséine $\kappa$ pendant les fabrications fromagères (Walstra et Jenness, 1992 ; Kumar et al., 2006). Cependant cette activité protéolytique se manifeste aussi sur toutes les protéines au cours de l'affinage du fromage.

\section{Les mécanismes de la coagulation par la présure}

La présure (préparation enzymatique coagulante) dans le lait libère le caséinomacropeptide (CMP) et induit une diminution de l'hydratation des micelles de caséine (passage de 2,5 g d'eau par gramme de caséine à 1,6 - 1,7 g/g à $\mathrm{pH} 6,60$, et de la charge nette des micelles de caséine (cette charge passe de $-15-20 \mathrm{mV}$ à $-5 \quad-8 \mathrm{mV}$ ) (Mietton et al., 2004).

Le mécanisme se déroule en trois phases principales (Payen, 1976; Visser, 1981; Green et Morant, 1981; Walstra et Jenness, 1992; Dalgleish, 1992).

\section{Phase primaire}

La présure attaque la composante qui stabilise la micelle de caséine (la caséine $\kappa$ ) au niveau de la liaison PHE105-MET106 (Mahaut et al., 2000 ; Vignola, 2002; Anema et al., 2005).

Il y a alors coupure de la chaîne peptidique en deux segments inégaux: le segment 1-105 est la paracaséine- $\kappa$ et le segment 106-169, le caséinomacropeptide (CMP). La paracaséine- $\kappa$ liée aux caséines $\alpha$ 
et $\beta$ reste intégrée à la micelle hydrophobe et le CMP contenant tous les glucides est libéré et passe dans le lactosérum. Le CMP est la partie de la caséine $\kappa$ qui est fortement chargée négativement et responsable des répulsions stériques et électrostatiques. La chymosine supprime cette chevelure stabilisante de la micelle (Cayot et Lorient, 1998).

\section{Phase secondaire}

C'est une étape d'agglomération de mécanisme encore mal connu. Lors de cette phase, les micelles déstabilisées peuvent se rapprocher et former des liens hydrophobes par intervention des ions calcium qui s'unissent à la partie chargée négativement des micelles diminuant ainsi les forces de répulsion électrostatiques auxquelles elles sont soumises ce qui favorise leur agrégation (Holt, 1997; Mietton et al., 2004). Cette phase est observable par la formation du gel. Le démarrage de cette phase nécessite qu'au moins 85 à $90 \%$ de la caséine $\kappa$ soit hydrolysée. Le CMP se détache de la caséine $\kappa$ et la micelle perd son caractère hydrophile. Il y a diminution de son degré d'hydratation et de son potentiel de surface. Des liaisons hydrophobes et électrostatiques s'établissent alors entre les micelles modifiées et vont entraîner la formation du gel (Dalgleish, 1992; Mahaut et al., 2000).

\section{Phase tertiaire}

Les micelles agrégées subissent de profondes réorganisations par la mise en place de liaisons phosphocalciques et peut-être des ponts disulfures entre les paracaséines. Ces mécanismes survenant au cours des phases (2 et 3) sont assez complexes et encore mal connus (Mahaut et al., 2000).

\section{Paramètres influant la coagulation présure}

Plusieurs facteurs influencent la coagulation présure: parmi ces facteurs, on peut citer la concentration en enzyme, la température, le $\mathrm{pH}$ et la concentration en ions
Ca 2+ (Larsson et Andren, 1999; Vliet et al., 2004; Candioti et al., 2004; Anema et al., 2005; Guyomarc, 2006).

\section{La concentration en enzyme}

Comme toute réaction enzymatique, la concentration en enzyme a une grande influence sur le processus de coagulation présure. Cette coagulation est le résultat d'une réaction d'hydrolyse enzymatique suivie d'une réaction d'agrégation des protéines (Van Hooydonk et Walstra, 1987). Le temps de coagulation diminue avec l'augmentation de la concentration en enzyme (McMahon et al., 1984a; Carson et al., 1987). Certains auteurs ont observé aussi une augmentation de la fermeté du gel associée à l'augmentation de la concentration en présure (Zoon et al., 1988).

\section{La température}

La coagulation enzymatique du lait est fortement dépendante de la température. La vitesse de formation du coagulum augmente progressivement de 20 à $40-42^{\circ} \mathrm{C}$

En dessous de $10{ }^{\circ} \mathrm{C}$, la gélification ne se produit pas. L'effet le plus important de la baisse de la température est la solubilisation du phosphate de calcium micellaire à une température plus élevée, le processus de coagulation ralentit. Il s'arrête à $55^{\circ} \mathrm{C}$.

Après un chauffage, il $\mathrm{y}$ a une augmentation du temps de gélification par la chymosine accompagnée d'une diminution de la fermeté du gel formé. D’après leur étude, le chauffage a un léger effet au niveau de la phase primaire (enzymatique) de la coagulation, tandis que son effet reste marqué au moment de la phase secondaire (phase d'agrégation des protéines) (Alloggi et al., 2000; Najera et al., 2003; Anema et al., 2005).

\section{Le $p H$}

L'influence du pH sur le temps de coagulation et la vitesse de raffermissement du caillé est très forte (Pires et al., 1999; 
Awad, 2005). Le pH optimal d'hydrolyse de la $\kappa$ - caséine se situe entre 5,1-5,3 (Humme, 1972; Lopez et al., 1998; Zbikowska et al., 2004) L'effet le plus important de l'abaissement du $\mathrm{pH}$ est la solubilisation du phosphate de calcium micellaire (Visser et al., 1980 ; Le Graet et Brule, 1993), ainsi que la diminution de la charge nette de la molécule de caséine, suivi de la dissociation de la caséine des micelles. L'abaissement du pH augmente la vitesse de raffermissement du caillé (Daviau et al., 2000).

\section{La concentration en ions $\mathrm{Ca}^{2+}$}

L'addition de calcium $\left(\mathrm{Ca}^{2+}\right)$ diminue le temps de coagulation par la présure (McMahon et al., 1984b; Moutilla et al., 1995; Balcones et al., 1996). Cette addition de $\mathrm{CaCl}_{2}$ au lait ou à une suspension micellaire entraîne un abaissement du niveau de protéolyse de la caséine $\kappa$ nécessaire à l'agrégation (Famelart, 2004). L'agrégation des caséines est ainsi accélérée par l'addition de calcium. Cette accélération est due à la réduction du potentiel $\zeta$ des micelles (Dalgleish, 1983; Holt, 1997). L'influence du calcium affecte aussi la vitesse de raffermissement ainsi que la fermeté du gel (Patel et Reuter, 1986 ; Lucey et Fox, 1993).

Le calcium apporté sous forme de $\mathrm{CaCl}_{2}$ entraîne une augmentation de la force ionique, une diminution de $\mathrm{pH}$ et une élévation du produit de solubilité apparent du phosphate de calcium. Il en résulte alors une augmentation des concentrations en calcium soluble et ionique. Le Chlorure de calcium «écrante» les charges positives et négatives des micelles et augmente leur hydratation (Zoon et al., 1989).

A des concentrations de $\mathrm{Ca}^{2+}$ élevées $(\geq$ $0,3 \mathrm{M})$, le temps de coagulation peut diminuer. Une addition de plus de $10 \mathrm{mM}$ de $\mathrm{Ca}^{2+}$ au lait augmente la force du gel présure (Lucey et Fox, 1993). Le couple $\mathrm{pH}$ et la concentration du lait en chlorure de calcium $\left(\mathrm{CaCl}_{2}\right)$ est la seule combinaison qui affecte la fermeté du coagulum et la vitesse de formation du gel (Najera et al., 2003; Mietton et al., 2004).



Figure 1: pourcentage des différentes protéines du lait (Cayot et Lorient, 1998 cités par Vignola, 2002). 


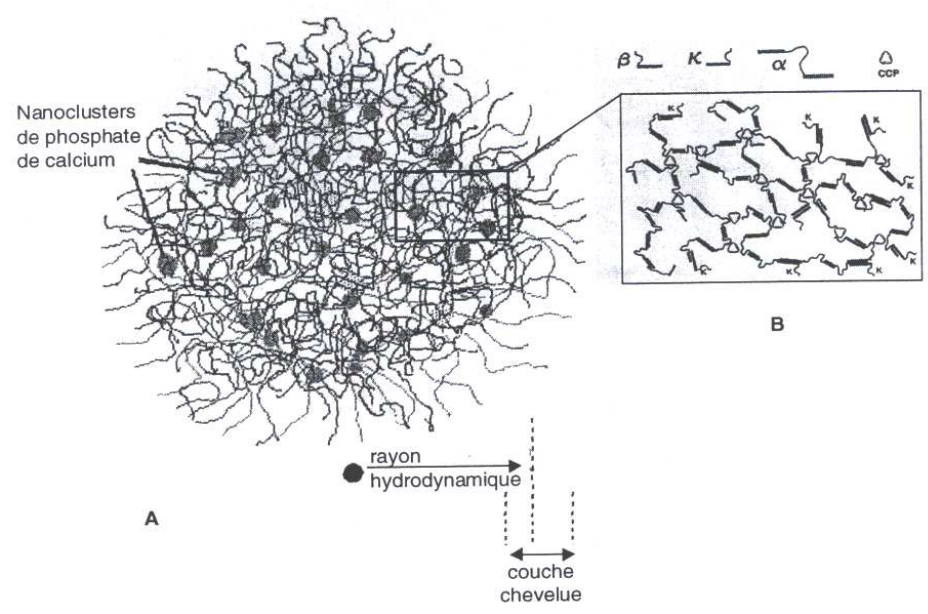

Figure 2: Modèle à structure ouverte de la micelle de caséine (Holt et Horne, 1996).

Tableau 1: Composition moyenne de la micelle de caséine en g/100g (Schmidt, 1980).

\begin{tabular}{llll}
\hline Caséines & \multicolumn{3}{c}{ Composants salins } \\
\hline$\alpha_{\mathrm{s} 1}$ & 33 & Calcium & 2,9 \\
$\alpha_{\mathrm{s} 2}$ & 11 & Magnésium & 0,2 \\
$\beta$ & 33 & Phosphate inorganique & 4,3 \\
$\kappa$ & 11 & Citrate & 0,5 \\
$\gamma$ & 4 & & \\
Total caséines & $\mathbf{9 2}$ & Total composants salins & $\mathbf{8 , 0}$ \\
\hline
\end{tabular}

\section{Conclusion}

Le processus de gélification du lait est sous la dépendance étroite de l'aptitude des protéines du lait particulièrement des caséines à floculer lorsque leur émulsion est soumise à l'action des enzymes coagulantes comme la présure. Ce phénomène a une importance capitale en technologie laitière particulièrement en technologie fromagère. Les différents travaux des scientifiques sur ce processus ont permis d'élucider le mécanisme biochimique de cette coagulation et les facteurs qui l'influencent en l'occurrence le pH du milieu, la température, la concentration en enzyme et la concentration en ions $\mathrm{Ca}^{2+}$. Ces connaissances ont permis aux professionnels de mieux maîtriser cette étape délicate de la coagulation et d'avoir de bons rendements de fabrication et de produits de qualité. Ainsi la technologie fromagère a atteint un niveau de technicité très élevé grâce aux connaissances acquises dans le domaine de la science du lait, sur les composants du lait, et sur leurs évolutions et leurs interactions au cours de la transformation.

\section{REFERENCES}

Alais C. 1984. Science du lait. Principes des Techniques. Edition Sepaic: Paris ; 814p.

Alloggi V, Caponio F, Pasqualone A, Gomes Tommaso. 2000. Effect of heat treatment on the rennet clotting time of goat and cow milk. Food Chemistry, 70: 51-55.

Amiot J, Fournier S, Lebeuf Y, Paquin P, Simpson R. 2002. Composition, propriétés physicochimiques, valeur 
nutritive, qualité technologique et techniques d'analyse du lait. Dans: Sciences et technologie du lait. Ecole Polytechnique de Montréal, 600p.

Anema SG, Lee SK, Klostermeyer H. 2005. Effect of $\mathrm{pH}$ at heat treatment on the hydrolysis of $\kappa$-casein and the gelation of skim milk by chymosin. LWT Food Science and Technology, 40: 99-106.

Areces LB, Biscoglio de Jimenez Bonino MJ, Parry MAA, Fraile ER, Fernandez HM, Cascome O. 1992. Purification and characterization of a milk clotting protease from Mucor baciliformis. Applied Biochemistry and Biotechnology, 37: 283-294.

Arima K, Iwasaki S, Tamura G. 1967. Agriculture Biology Chemistry, 31: 540543.

Arima K, Yu J, Iwasaki S. 1970. Milk-clotting enzyme from Mucor pusillus var.Lindt. Methods in Enzymology, 19: 446-459.

Awad S. 2005. Effect of sodium chloride and $\mathrm{pH}$ on the rennet coagulation and gel firmness. $L W T$ - Food Science and Technology, 40: 220-224.

Aworh OC and Muller HG. 1987. Cheesemaking properties of vegetable rennet from Sodom apple (Calotropis procera). Food Chemistry, 26: 71-79.

Balcones E, Olano A, Calvo MM. 1996. Factors affecting the rennet clotting properties of ewe's milk. Journal of Agriculture and Food Chemistry, 44: 1993-1996.

Bonfatti V, Martino G , Cecchinato A , Degano L, Carnier P. 2010. Effects of beta Kappa casein (CSN2-CSN3) haplotypes, beta-lactoglobulin $(B L G)$ genotypes, and detailed protein composition on coagulation properties of individual milk of Simmental cows. Journal of Dairy Science, 93(8): 38093817.

Brulé G, Lenoir J, Reneuf F. 1997. La micelle de caséine et la coagulation du lait. In le Fromage. TEC \& DOC Lavoisier: Paris; $7-41$.
Carson A, Hill C, Olson NF. 1987. Kinetics of milk coagulation: III mathematical modelling of the kinetics of curd formation following enzymatic hydrolysis of kappa-casein-parameter estimation. Biotechnology and Bioengineering, 29: 601-611.

Cavalcanti MTH, Teixera MFS, Lima Filho JL, Porto ALF. 2004. Partial purification of new milk-clotting enzyme produced by Nocardiopsis sp. Bioresource Technology, 93: 29-35.

Cayot P, Lorient D. 1998. Structures et Technofonctions des Protéines du Lait. TEC \& DOC Lavoisier: Paris; 363 p.

Dalgleish DG. 1983. Coagulation of renneted bovine casein micelles: dependence On temperature, calcium ion, concentration and ionic strength. Journal of Dairy Research, 50: 331-340.

Dalgleish DG, Law JR. 1989. pH-induced dissociation of bovine casein micelles. II. Mineral solubilization and its relation to casein release. Journal of Dairy Research, 56: 727-735.

Dalgleish DG. 1992. The enzymatic coagulation of milk. Advanced Dairy Chemistry Proteins, 1: 579-619.

Dalgleish DG. 1998. Casein micelles as colloids: surface structures and stabilities. Journal of Dairy Science, 81: 3013-3018.

D'Ambrosio A, Rossano R, Uungaro N, and Riccio P. 2003. Proteolytic and milk clotting activities in extracts obtained from crustaceans Munida. Journal of Molecular Catalysis B: Enzymatic, 22: 145-150.

Daviau, C, Famelart MH, Pierre A, Goudedranche H, Mauboi JL. 2000. Rennet coagulation of skim milk and curd drainage : effect of $\mathrm{pH}$, casein concentration, ionic strength and heat treatment. Lait, 80: 397-415.

Chandrapala J, Augustin MA, McKinnon I, Udabage P. 2010. Effects of $\mathrm{pH}$, calcium-complexing agents and milk solids concentration on formation of soluble protein 
aggregates in heated reconstituted skim milk. International Dairy Journal, 20(11): 777-784.

Dickson IR, Perkins DJ. 1971. Studies on the interactions between purified bovine caseins and alkaline-earth-metalions. Biochemistry Journal, 124: 235-240.

Famelart MH. 2004. Environnement minéral et propriétés de gélification des caséines. In Minéraux et Produits Laitiers. TEC \& DOC Lavoisier: Paris; 585-613.

Farrell HM, Jr Brown EM, Kumosinski TF. 1993. Three Dimensional molecular modeling of bovine caseins. Food Structure, 12: 235-250.

Fernandez FM, Sanchez MH, Bigliardo GR. 2009. Comparative aspects of Milk caseins. Opera Lilloana, 47: 186.

Ghazarossian HAZAROSSIAN F. 1996. Etude de l'effet des hautes pressions sur la structure de la micelle de caséine bovine. Mémoire d'ingénieur, ENSBANA. Université de Bourgogne France.

Green ML, Morant SV. 1981. Mechanism of aggregation of casein micelles in rennettreated milk. Journal of Dairy Reearchs, 48(1): 57-63.

Guyomarc HF. 2006. Formation of heatinduced protein aggregates in milk as a means to recover the whey protein fraction in cheese manufacture, and potential of heat-treating milk at alkaline $\mathrm{pH}$ values in order to keep its rennet coagulation properties. A review. Lait, 86(1): 1-20.

Hallen E, Lunden A, Allmere T, Andren A. 2010. Casein retention in curd and loss of casein into whey at chymosin-induced coagulation of milk. Journal of Dairy Research, 77(1): 71-76.

Hemar Y, Singh H, Horne DS. 2004. Determination of early stages of rennetinduced aggregation of casein micelles by diffusing wave spectroscopy and rheological measurements. Current Applied Physics, 4: 362-365.
Holt C, Horne DS. 1996. The dairy casein micelle: evolution of the concept and its implications for dairy technology. Netherlands Milk and Dairy Journal, 50: 85-111.

Holt C. 1997. The milk salts and their interaction with caseins. In Advanced in Dairy Chemistry: Lactose, Water, Salts and Vitamins (Vol. 3). Chapman \& Hall: London; 233-256.

Holt C. 1992. Structure and stability of bovine casein micelles. Advances in Protein Chemistry, 43: 63-151.

Holt C, Horne DS. 1998. Casein interactions: casting light on the black boxes, the structure in dairy products. International Dairy Journal, 8: 171-177.

Humme HE. 1972. The optimum $\mathrm{pH}$ for the limited specific proteolysis of $\kappa$-caséine by rennin (primary phase of milk clotting). Netherlands Milk Dairy Journal, 26: 180-158.

Jean K, Renan M, Famelart MH, Guyomarc'h F. 2006. Structure and surface properties of the serum heat-induced protein aggregates isolated from heated skim milk. International Dairy Journal, 16(4): 303-315.

Kees Marlies. 1996. Le fromage peulh: facile à produire et bien apprécié. Une technologie à vulgariser. Rapport de Recherche /GTZ, pp.12-17.

Kethireddipalli P, Hill AR, Dalgleish DG. 2010. Protein interactions in heat-treated milk and effect on rennet coagulation. International Dairy Journal, 20(12): 838843.

Kumar A, Sharma J, Mohanty AK, Grover S and Batish VK. 2006. Purification and characterization of milk clotting enzyme from goat (Capra hircus). Comparative Biochemistry and Physiology Part B: Biochemistry and Molecular Biology, 145: 108-113.

Larsson KI, Andren A. 1999. Interactions between chymosin and individual or 
micellar caseins. International Dairy Journal, 9: 381-382.

Le Graet Y, Brule G. 1993. Les équilibres minéraux du lait: influence du pH et de la force ionique. Lait, 73: 51-60.

Little M, Holt C. 2004. An equilibrium thermodynamic model of the sequestration of calcium phosphate by casein phosphopeptides. European Biophysics Journal with Biophysics Letters, 33: 435-447.

Lopez MB, Lomholt SB, Qvist KB. 1998. Rheological properties and cutting time of rennet gels. Effects of $\mathrm{pH}$ and enzyme concentration. International Dairy Journal, 8: 289-293.

Lucey JA, Fox PF. 1993. Importance of calcium and phosphate in cheese manufacture: a review. Journal of Dairy Science, 76: 1714-1724.

Mahaut M, Jeantet R, Brule G. 2000. Initiation à la Technologie Fromagère. TEC \& DOC Lavoisier : Paris ; 194 p.

Martin P, Raymond MN, Bricas E, RibadeauDumas B.1980. Kinetic studies on the action of Mucor pusillus, Mucor meihei acid protease and chymosins $\mathrm{A}$ and $\mathrm{B}$ on a synthetic chromophoric hexapeptide. Biochemica Biophysica Acta, 612: 410420.

Mathieu H. 1985. Facteurs de variation de la composition du lait. In Lait et Produits Laitiers. 1. Les Laits de la Mamelle à la Laiterie. TEC \& DOC Lavoisier: Paris; 119-177.

McGann TCA, Donnely WJ, Kearney RD, Buccheim W. 1980. Composition and size distribution of bovine casein micelles. Biochimica et Biophysica Acta, 630: $261-270$.

McMahon DJ, Brown RJ, Ernstrom CA. 1984a. Enzymatic coagulation of milk casein micelles. Journal of Dairy Science, 67: 745-748.

McMahon DJ, Brown RJ, Richardson GH, Ernstrom CA. 1984b. Effects of calcium, phosphate and bulk culture media on milk coagulation properties. Journal of Dairy

Science, 67: 930-938.

McMahon DJ, McManus WR. 1998. Rethinking casein micelle structure using electron microscopy. Journal of Dairy Science, 81: 2985-2993.

McNeill GP, Donnely WJ. 1987. Optimization of porous glass, chromatography for sizefractionation of bovine casein micelles. Journal of Dairy Research, 54: 19-28.

Mercier JC, Brignon G, Ribardeau-Dumas B. 1973. Structure primaire, de la caséine $\kappa$ B bovine. Séquence complète. European Journal of Biochemistry, 35: 222-235.

Mietton B, Gaucheron F, Salaun F. 2004. Minéraux et transformations fromagères. In Minéraux et Produits Laitiers, GAUCHERON F (ed). Lavoisier: Paris; 471-563.

Moutilla A, Balcones E, Olano A, Calvo MM. 1995. Influence of heat treatments on whey protein denaturation and rennet clotting properties of cow's and goat's milk. Journal of Agriculture and Food Chemistry, 43: 1908-1911.

Najera AI, de Renobales M, Barron LJR. 2003. Effects of $\mathrm{pH}$, temperature, $\mathrm{CaCl} 2$ and enzyme concentrations on the rennetclotting properties of milk: a multifactorial study. Food Chemistry, 80: 345-352.

Patel RS, Reuter H. 1986. Effect of sodium, calcium and phosphate on Properties of rennet coagulated milk. Lebensmittel Wissenschaft Technologie, 19: 288-291.

Payen TAJ. 1976. Netherlands Milk and Dairy Journal, 30: 55-59.

Pires MS, Orellana GA, Gatti CA. 1999. Rennet coagulation of casein micelles and heated casein micelles: action of $\mathrm{Ca}^{2+}$ and pH. Food Hydrocolloids, 13: 235-238.

Pyne GT, McGann TCA. 1960. The colloidal phosphate of milk II. Influence of citrate. Journal of Dairy Research, 27: 9-17.

Remeuf F, Cossin V, Dervin C, Lenoir J, Tomassone R. 1991. Relation entre les caractères physicochimiques des laits et 
leur aptitude fromagère. Lait, 71: 397421.

Ribadeau-Dumas B, Grappin R. 1989. Milk protein analysis. Lait, 69: 357- 416.

Sardinas JL.1968. Rennin enzyme of Endothia parasitica. Applied Microbiology, 16: 248-255.

Schmidt DG. 1980. Colloidal aspects of casein. Netherlands Milk and Dairy Journal, 34: 42-64.

Schmidt DG. 1982. Association of caseins and casein micelle structure. In Developments in Dairy Chemistry. Elsevier: London; 61-86.

Singh H, Waungana A. 2001. Influence of heat treatment of milk on Cheese making properties. International Dairy Journal, 11: 543-551.

Swaisgood HE. 1982. Chemistry of milk protein. In Developments in Dairy Chemistry. Applied Science Publishers: London and New York; 1-59.

Van Dijk HJM. 1990. The properties of casein micelles.1. The nature of the micellar calcium phosphate. Netherlands Milk and Dairy Journal, 44: 65-81.

Van Hooydonk ACM, Walstra P. 1987. Interpretation of the kinetics of The renneting reaction in milk. Netherlands Milk Dairy Journal, 41: 19-47.

Vignola CL. 2002. Science et Technologie du Lait. Transformation du Lait. Fondation et Technologie Laitière du Québec. Presses Internationales Polytechnique: Québec; 600 p.

Visser S, Van Rooyen PJ, Slangen CJ. 1980. Peptide substrates for chymosin (rennin). Isolation and substrate behaviour of two tryptic fragments of bovine $\kappa$-casein.
European Journal of Biochemistry, 108: 415-421.

Visser S. 1981. Proteolytic enzymes and their action on milk proteins. Neth. Milk Dairy J., 35: 65-88.

Vliet TV, Lakemond CMM and Visschers RW. 2004. Rheology and Structure of milk protein gel. Current opinion in Colloid \& Interface Science, 9: 298-304.

Walstra P and Jenness R. 1992. Casein micelles. In Dairy Chemistry and Physics. John: New York; 229-253.

Walstra P. 1999. Casein sub-micelles: do they exist? International Dairy Journal, 9:189-192.

Walstra P, Geurts TJ, Noomen A, Jellema A, van Boekel MAJS. 1999. Principles of Milk Properties and Process, Dairy Technology. In Food Science and Technology, Bekker M (ed). Wageningen Agricultural, Univ New York (USA): New York; 727p.

Zbikowska A, Szerszunowicz I, Smyk B. 2004. Effect of $\mathrm{pH}$ on the composition and surface hydrophobicity of proteins forming the gel matrix during enzymatic coagulation of heated milk reconstituted from nonfat dry milk. Milchwissenschaft, 59(7/8): 417-420.

Zoon P, Van Vliett T, Walstra P. 1989. Rheological properties of rennet- induced skim milk-gels. IV: The effect of $\mathrm{pH}$ and $\mathrm{NaCl}$. Netherlands Milk and Dairy Journal, 43: 17-34.

Zoon P, Van Vliet T, Walstra P. 1988. Rheological properties of rennet- induced skim milk-gels. 1. Introduction. Netherlands Milk and Dairy Journal, 42: 249-269. 